\title{
WEIGHTED COMPOSITION OPERATORS WITH CLOSED RANGE
}

\section{N. Palmberg}

\begin{abstract}
We study the closed range property of weighted composition operators on weighted Bergman spaces of infinite order (including the Hardy space of infinite order). We give some necessary and sufficient conditions and find a complete characterisation for weighted composition operators associated with conformal mappings. We also give the corresponding results for composition operators on the Bloch-type spaces. Therefore, the results obtained in this paper also improve and generalise the results of Ghatage, Yan, Zheng and Zorboska.
\end{abstract}

\section{INTRODUCTION}

We denote by $H(\mathbb{D})$ the space of holomorphic functions on the unit disk $\mathbb{D}$. Throughout the paper $\varphi \in H(\mathbb{D})$ will denote a non-constant function satisfying $\varphi(\mathbb{D}) \subseteq \mathbb{D}$, while $\psi \in H(\mathbb{D})$ will be any function not identically equal to zero. The weighted composition operator $W_{\varphi, \psi}$, induced by $\varphi$ and $\psi$, is the linear map on $H(\mathbb{D})$ defined by $W_{\varphi, \psi} f=\psi \cdot(f \circ \varphi)$. Thus, the results obtained in this paper also hold for the classical composition operator $C_{\varphi}$ and the multiplication operator $M_{\psi}$, which we get by choosing $\psi \equiv 1$ and $\varphi(z)=z$, respectively. In fact, $W_{\varphi, \psi}=M_{\psi} C_{\varphi}$. Various properties of composition operators, multiplication operators and weighted composition operators have been intensively studied in many papers (see for example $[2,3,4,5,8,17,20,21,23,25]$ ). A good general reference on composition operators is the book by Cowen and MacCluer [9]. In this paper we study the closed range property, which has been the main object of investigation in several papers recently (see for example $[7,13,14]$ as well as the related references therein).

The Banach spaces that we shall consider are the weighted Bergman spaces of infinite order,

$$
H_{v}^{\infty}:=\left\{f \in H(\mathbb{D}):\|f\|_{v}=\sup _{z \in \mathbf{D}}|f(z)| v(z)<\infty\right\}
$$

Received 20th June, 2006

The research was partially supported by the Academy of Finland Project No. 205644.

I wish to thank my advisor Mikael Lindström for pointing out these problems to me and for many helpful discussions. I would also like to thank Pawel Domański and Kinga Cichon for suggesting and providing me with some useful references.

Copyright Clearance Centre, Inc. Serial-fee code: 0004-9727/07 \$A2.00+0.00. 
where $v: \mathbb{D} \rightarrow \mathbb{R}_{+}$is a radial continuous function such that $H_{v}^{\infty}$ contains a non-zero function (see Section 2 for more details). We endow them with the norm $\|\cdot\|_{v}$. These spaces occur in various settings in for example $[3,4,5,7,8,10,19]$. See also the survey of operators between these spaces by Bonet [2] as well as the references therein. We shall give our results with as few restrictions on the weights as possible (except for some general assumptions). On many occasions, the weights given by

$$
v_{q}(z):=\left(1-|z|^{2}\right)^{q}, \quad \text { where } 0<q<\infty,
$$

will come in handy while proving results for more general weights (see [16] for a thorough treatment of the $H_{v_{q}}^{\infty}$-spaces). In Section 3 we concentrate on the $H_{v}^{\infty}$-spaces, where some of the results are directly applicable to $H^{\infty}$ (that is, the classical Hardy space of infinite order endowed with the usual supremum norm $\left.\|\cdot\|_{\infty}\right)$. In Section 4 we study weighted composition operators associated with conformal mappings, obtained by choosing $\varphi$ univalent and $\psi=\left(\varphi^{\prime}\right)^{\mu}$, with $\mu>0$. In this case we give a complete characterisation of the closed range property. These operators have recently been studied in for example [24]. In Section 5 we take a more concrete look at our results by giving some enlightening examples. Finally, in Section 6 we apply our results from the preceding sections to composition operators on the Bloch-type spaces,

$$
\mathcal{B}^{q}:=\left\{f \in H(\mathbb{D}):\|f\|_{\mathcal{B}^{q}}=\sup _{z \in \mathbb{D}}\left|f^{\prime}(z)\right|\left(1-|z|^{2}\right)^{q}<\infty\right\}
$$

where $0<q<\infty$. These become Banach spaces when we identify functions differing by a constant and endow them with the norm $\|\cdot\|_{B^{a}}$ (see $[8,20]$ for more on these spaces). Note that for $q=1$ we get the classical Bloch space $\mathcal{B}$ and for $0<q<1$, we have the analytic Lipschitz spaces of order $1-q$ (see [9, Theorem 4.1]). The main results in $[13,14]$ become a direct consequence of the results in Section 6 .

\section{PReliminaries AND notations}

Throughout the paper we shall say that $A \approx B$ if there are strictly positive constants $c_{1}$ and $c_{2}$, such that $c_{1} A \leqslant B \leqslant c_{2} A$, where the constants don't depend on properties of $A$ and $B$. Furthermore, $\partial \mathbb{D}$ will denote the boundary of the unit disk, while the constants $\mu$ and $q$ (as well as $q_{1}$ and $q_{2}$ ) will always be strictly positive constants. Finally, $B_{H_{v}^{\infty}}$ will denote the closed unit ball of $H_{v}^{\infty}$, while $d A$ will denote the Lebesgue area measure on the plane normalised so that $A(\mathbb{D})=1$.

THE METRICs. For the results in this subsection, [12] serves as a general reference (see also [28, Section 4.3]). The pseudo-hyperbolic metric is given by

$$
\rho(z, a):=\left|\sigma_{a}(z)\right|, \quad \text { where } \quad \sigma_{a}(z):=\frac{a-z}{1-\bar{a} z}
$$


is the automorphism of $\mathbb{D}$ that changes 0 and $a$. One of the most important properties of the pseudo-hyperbolic metric is that it is Möbius invariant, or more precisely, that $\rho(\sigma(z), \sigma(a))=\rho(z, a)$ for all automorphisms $\sigma$ of $\mathbb{D}$. Furthermore, we shall frequently use the following equalities which are both easy to check:

$$
1-\left|\sigma_{a}(z)\right|^{2}=\frac{\left(1-|a|^{2}\right)\left(1-|z|^{2}\right)}{|1-\bar{a} z|^{2}}=\left|\sigma_{a}^{\prime}(z)\right|\left(1-|z|^{2}\right) .
$$

For any $a \in \mathbb{D}$ and $r \in(0,1], D(a, r)$ denotes the pseudo-hyperbolic disc with centre $a$ and radius $r$ (normalised so that $|D(0,1)|=1$ ). That is,

$$
D(a, r):=\left\{z \in \mathbb{D}:\left|\sigma_{a}(z)\right|<r\right\} .
$$

$D(a, r)$ is an Euclidean disk with centre $c_{E}(a, r)$ and radius $r_{E}(a, r)$ given by

$$
c_{E}(a, r)=\frac{\left(1-r^{2}\right) a}{1-r^{2}|a|^{2}} \quad \text { and } \quad r_{E}(a, r)=\frac{\left(1-|a|^{2}\right) r}{1-r^{2}|a|^{2}}
$$

The pseudo-hyperbolic metric is a true metric. In fact, it even satisfies a stronger version of the triangle inequality, stating that for any $z, a, b \in \mathbb{D}$, we have that

$$
\rho(z, a) \leqslant \frac{\rho(z, b)+\rho(b, a)}{1+\rho(z, b) \rho(b, a)} .
$$

The standard Euclidean metric is given by $\operatorname{dist}(z, a):=|z-a|$, while the Bergman metric (also called the hyperbolic or the Poincare metric) is given by

$$
\beta(z, a):=\frac{1}{2} \log \frac{1+\rho(z, a)}{1-\rho(z, a)} .
$$

If $d s$ is the Euclidean element of length, then the length element in the Bergman metric is given by $\left(1-|z|^{2}\right)^{-1} d s$. Naturally, the Bergman metric is also Möbius invariant.

THE WEIGHTS. A weight is a continuous and strictly positive function on $\mathbb{D}$, which is radial (that is, $v(z)=v(|z|))$. Recall that a real-valued function $f$ is said to be almost decreasing if there exists a strictly positive constant $c$ such that $f(x) \geqslant c f(y)$ whenever $x \leqslant y$. We shall say that the weight $v$ is typical if it is almost decreasing and $\lim _{|z| \rightarrow 1} v(z)=0$. It is worth mentioning that when $v$ is a bounded weight with $\lim _{|z| \rightarrow 1} v(z)>0$, then $H_{v}^{\infty}$ is isometrically isomorphic to $H^{\infty}$. When dealing with weighted spaces of analytic functions one often stumbles across the so-called associated weights. These weights are given by

$$
\tilde{v}(z):=\left(\sup _{f \in B_{H \infty}}|f(z)|\right)^{-1} .
$$

It is well known that $H_{\tilde{v}}^{\infty}$ is isometrically isomorphic to $H_{v}^{\infty}$. Since $v(z)$ is always less than or equal to $\widetilde{v}(z)$, we get that $v \approx \widetilde{v}$ if there is a positive constant $c$ such that 
$\tilde{v}(z) \leqslant c v(z)$. In this case we say that the weight $v$ is essential. All these definitions and facts on associated weights can be found in [1] (see also [5]).

A moderate weight $v$ is a function in $C^{2}(\mathbb{D})$, satisfying

$$
-\Delta \log v(z) \approx \frac{1}{\left(1-|z|^{2}\right)^{2}} \quad \text { near } \partial \mathbb{D}, \quad \text { where } \quad \Delta:=\frac{1}{4}\left(\partial_{|z|}^{2}+\frac{1}{|z|} \partial_{|z|}\right)
$$

is the standard Laplacian divided by a constant of 4 . See [10] and the related references therein for a background on moderate weights. Lusky studied in [19] weights satisfying the conditions $L_{1}$ and $L_{2}$ (renamed here after the author) given by

$L_{1}$

$$
\inf _{n \in \mathbb{N}} \frac{v\left(1-2^{-n-1}\right)}{v\left(1-2^{-n}\right)}>0
$$

$L_{2}$

$$
\limsup _{n \in \mathbb{N}} \frac{v\left(1-2^{-n-j}\right)}{v\left(1-2^{-n}\right)}<1 \quad \text { for some } j \in \mathbb{N} \text {. }
$$

Such weights are called normal by [26] (see also [27]). An almost decreasing weight $v$ satisfies condition $L_{1}$ if and only if there exists a (large enough) constant $q_{1}$ such that $v_{q_{1}} / v$ is almost decreasing. Similarly, it satisfies condition $L_{2}$ if and only if there exists a (small enough) constant $q_{2}$ such that $v / v_{q_{2}}$ is almost decreasing (see Lemma 1 and the proof thereof in [10]). In other words, condition $L_{1}$ is another way of saying that the weight $v$ shouldn't tend to zero too fast. Condition $L_{2}$ on the other hand says that it shouldn't tend to zero too slowly either. Actually, every moderate weight is normal and for every almost decreasing normal weight, we can find an equivalent weight which is moderate (see [10, Lemma 1 and Theorem 10] as well as [4, p. 185]). Let

$$
V_{m}:=\{v: v \text { is a moderate weight }\},
$$

which on account of what was just said, incorporates in some sense the normal weights. Concrete examples of weights satisfying condition $L_{1}, L_{2}$ or both can be found in $[4,7$, $10,19]$ (see also $[26,27]$ ).

Finally, we make the useful observation that $\tilde{v}$ is always non-increasing due to the Hadamard three circle theorem (see [28, Theorem 2.2.2]). This on the other hand implies that all essential weights are almost decreasing. Also notice that all moderate weights are typical (and essential) and that the weight $v_{q}$ is a moderate weight which actually satisfies $\tilde{v_{q}}=v_{q}$.

THE OPERATORS. We shall only concern ourselves with bounded operators, so naturally we need to know when a weighted composition operator is bounded on $H_{v}^{\infty}$. By [8, Proposition 3.1] we have that

$$
W_{\varphi, \psi} \text { is bounded on } H_{v}^{\infty} \text { if and only if } \sup _{z \in \mathbb{D}} \frac{|\psi(z)| v(z)}{\widetilde{v}(\varphi(z))}<\infty .
$$


Moreover, by [8, Theorem 3.4] we know that $W_{\varphi, \psi}$ is bounded on $H_{v}^{\infty}$ for all almost decreasing weights if and only if $\psi \in H^{\infty}$ and there is $r_{0}>0$ such that $|\varphi(z)| \leqslant|z|$ for $|z| \geqslant r_{0}$. In $[8]$ they assume the weights to be non-increasing, but in Proposition 3.1 it is not needed and in Theorem 3.4 it can be replaced by almost decreasing. We shall use both these results without any further reference.

It is worth pointing out that since $\varphi$ is non-constant, the open mapping theorem for analytic functions ensures that $C_{\varphi}$ is one-to-one. Similarly, since $\psi$ is not identically equal to zero, $M_{\psi}$ is also one-to-one. Thus, $W_{\varphi, \psi}$ is one-to-one. Moreover, it has closed range if and only if it is bounded below. That is, if there exists $\varepsilon>0$ such that

$$
\left\|W_{\varphi, \psi} f\right\|_{v} \geqslant \varepsilon\|f\|_{v} \text { for all } f \in H_{v}^{\infty} .
$$

THE SET CONDITIONS. We say that the set $K \subseteq \mathbb{D}$ satisfies the reverse Carleson condition (see $[18,14]$ ) if there are constants $r \in(0,1)$ and $c \in(0,1)$ such that

$$
|D(a, r) \cap K| \geqslant c|D(a, r)| \text { for all } a \in \mathbb{D} .
$$

We shall use the following notations throughout the paper. For $\varepsilon>0$,

$$
{ }^{\varepsilon} \Omega_{\varphi, \psi}^{v}:=\left\{z \in \mathbb{D}:\left|\tau_{\varphi, \psi}^{v}(z)\right| \geqslant \varepsilon\right\}, \quad \text { where } \quad \tau_{\varphi, \psi}^{v}(z):=\frac{\psi(z) v(z)}{\widetilde{v}(\varphi(z))}
$$

(compare this to the characterisation of boundedness above). We will always assume that $\varepsilon$ is chosen so that the set ${ }^{\varepsilon} \Omega_{\varphi, \psi}^{v}$ is non-empty. In the special cases when $v \equiv 1$ or when $\varphi(z)=z$ and $v$ is essential, we simply write ${ }^{\varepsilon} \Omega_{\psi}$. Finally,

$$
T_{\varphi, \psi}^{v}:=\sup _{z \in \mathbb{D}}\left|\tau_{\varphi, \psi}^{v}(z)\right|
$$

\section{WEIGHTED BERGMAN SPACES OF INFINITE ORDER}

Multiplication operators with closed range has the following characterisation (see [4, Theorem 3.6 and Lemma 3.7]). $M_{\psi}$ has closed range on $H_{v}^{\infty}$ (where $v \in V_{m}$ and $\psi \in H^{\infty}$ ) if and only if for all $r \in(0,1)$ there exists $\varepsilon>0$ such that

$$
{ }^{\varepsilon} \Omega_{\psi} \cap \overline{D(a, r)} \neq \emptyset \text { for all } a \in \mathbb{D} \text {. }
$$

There are also other characterisations in [4] in terms of Gelfand transforms and Shilov boundaries, but in this paper we shall only present function theoretical results. The aim of this section is to give similar conditions for weighted composition operators. Some of the techniques we use are inspired by the ones used in $[13,14]$. Before we state the main theorem of this section, we shall prove an easy, but important lemma, which will simplify many things. We state it formally for completeness. 
LEMMA 3.1. Let $\phi=\sigma_{\varphi(0)} \circ \varphi$ and assume that the weight $\tilde{v}$ satisfies condition $L_{1}$. Then $W_{\varphi, \psi}: H_{v}^{\infty} \rightarrow H_{v}^{\infty}$ has closed range if and only if $W_{\phi, \psi}: H_{v}^{\infty} \rightarrow H_{v}^{\infty}$ has closed range. In other words, under this condition we may assume that $\varphi(0)=0$.

ProOF: By [5, Theorem 2.3], $\widetilde{v}$ satisfies condition $L_{1}$ if and only if for all $a \in \mathbb{D}$, the operator $C_{\sigma_{a}}$ is bounded on $H_{v}^{\infty}$. In [5] $v$ is assumed to be typical, but the same proof works without this assumption. Since $C_{\sigma_{a}}^{-1}=C_{\sigma_{a}}$, a straightforward computation finishes the proof.

THEOREM 3.2. (Moderate weights) Let $v \in V_{\mathrm{m}}$ and $\psi \in H^{\infty}$.

(a) (Necessity) If $W_{\varphi, \psi}$ has closed range on $H_{v}^{\infty}$, then for every $w \in V_{\mathrm{m}}$ there exists $\varepsilon>0$ such that

$$
\varphi\left({ }^{\varepsilon} \Omega_{\varphi, \psi}^{w}\right) \cap \overline{D(a, 1-\varepsilon)} \neq \emptyset \quad \text { for all } a \in \mathbb{D} .
$$

(b) (Sufficiency) Let $R_{q}$ be the constant given by

$$
R_{q}:=\frac{1}{2}\left(2 q+\frac{(2(q+1))^{q+1}}{q^{q}}+\left(\left(2 q+\frac{(2(q+1))^{q+1}}{q^{q}}\right)^{2}+16\right)^{1 / 2}\right),
$$

where $q>\limsup _{|z| \rightarrow 1}\left(1-|z|^{2}\right)^{2}(-\Delta \log v(z))$. If for any $w \in V_{\mathrm{m}}$ there are $r \in\left(0, R_{q}^{-1}\right)$ and $\varepsilon>0$ such that

$$
\varphi\left({ }^{\varepsilon} \Omega_{\varphi, \psi}^{w}\right) \cap \overline{D(a, r)} \neq \emptyset \quad \text { for all } a \in \mathbb{D},
$$

then $W_{\varphi, \psi}$ has closed range on $H_{v}^{\infty}$.

REMARK 3.3. Since $R_{q} \in(1+\sqrt{5}, \infty)$ is strictly (and rapidly) increasing as $q$ is increasing we want to choose $q$ as small as possible.

In order to prove Theorem 3.2 we shall need three lemmas and Theorem 3.7. The first lemma is a generalisation of [4, Lemma 3.1], the second one shows an useful fact about the sets ${ }^{\varepsilon} \Omega_{\varphi, \psi}^{v}$, while the third one is needed for the proof of Theorem 3.7.

LEMMA 3.4. Assume that $\varphi(0)=0$ and that the weights $v$ and $w$ are such that $u:=v / w$ is equivalent to an essential weight. Then $W_{\varphi, \psi}: H_{w}^{\infty} \rightarrow H_{w}^{\infty}$ has closed range whenever $W_{\varphi, \psi}: H_{v}^{\infty} \rightarrow H_{v}^{\infty}$ has closed range.

Proof: Assume that $W_{\varphi, \psi}$ is not bounded below on $H_{w}^{\infty}$. Then there exists a sequence $\left\{f_{n}\right\} \subseteq H_{w}^{\infty}$ (with $\left\|f_{n}\right\|_{w}=1$ ) such that $\left\|W_{\varphi, \psi} f_{n}\right\|_{w} \leqslant 1 / n$. Therefore, we can find a sequence $\left\{z_{n}\right\} \subseteq \mathbb{D}$ such that $\left|f_{n}\left(z_{n}\right)\right| w\left(z_{n}\right) \geqslant 1 / 2$. Furthermore, using the fact that the norm topology of $H_{v}^{\infty}$ is finer than the compact-open topology, we know that for all $z_{n} \in \mathbb{D}$ there exists $g_{n} \in B_{H_{s}^{\infty}}$ such that $\left|g_{n}\left(z_{n}\right)\right|=\widetilde{u}\left(z_{n}\right)^{-1}$. Hence, using the essentiality of $u$, we get that $\left|g_{n}\left(z_{n}\right)\right| u\left(z_{n}\right) \geqslant c>0$. Consider now the sequence $\left\{f_{n} g_{n}\right\}$. One can easily check that it belongs to $B_{H_{v}^{\infty}}$ and that $\left\|f_{n} g_{n}\right\|_{v} \geqslant c / 2$. Since $u$ is essential 
it is also almost decreasing. Thus, using Schwarz' lemma, there exists $c_{u}>0$ such that $u(z) \leqslant c_{u} u(\varphi(z))$. Hence,

$$
\begin{aligned}
\left\|W_{\varphi, \psi} f_{n} g_{n}\right\|_{v} & \leqslant \sup _{z \in \mathbb{D}}\left|f_{n}(\varphi(z))\right||\psi(z)| w(z) c_{u}\left|g_{n}(\varphi(z))\right| u(\varphi(z)) \\
& \leqslant\left\|W_{\varphi, \psi} f_{n}\right\|_{w} c_{u}\left\|g_{n}\right\|_{u} \\
& \leqslant \frac{c_{u}}{n},
\end{aligned}
$$

Clearly $W_{\varphi, \psi}$ cannot be bounded below on $H_{v}^{\infty}$.

Lemma 3.5. Assume that $\varphi(0)=0$ and that $\psi \in H^{\infty}$. If for some $v \in V_{\mathrm{m}}$ there exists $\varepsilon>0$ such that $\varphi\left({ }^{\varepsilon} \Omega_{\varphi, \psi}^{v}\right) \cap \overline{D(a, 1-\varepsilon)} \neq \emptyset$ for all $a \in \mathbb{D}$, then for all $w \in V_{\mathrm{m}}$ there exists $\delta>0$ such that $\varphi\left({ }^{\delta} \Omega_{\varphi, \psi}^{w}\right) \cap \overline{D(a, 1-\delta)} \neq \emptyset$ for all $a \in \mathbb{D}$.

Proof: Assume that $v \in V_{m}$ and that there exists $\varepsilon>0$ such that the intersection of $\varphi\left({ }^{\varepsilon} \Omega_{\varphi, \psi}^{v}\right)$ and $\overline{D(a, 1-\varepsilon)}$ is non-empty for all $a \in \mathbb{D}$. Take $w \in V_{m}$. We claim that we can find $q_{1}$ such that $w_{1}:=v_{q_{1}} / w$ is an essential weight. Indeed,

$$
-\Delta \log \frac{v_{q_{1}}(z)}{w(z)}=\frac{q_{1}}{\left(1-|z|^{2}\right)^{2}}-(-\Delta \log w(z))>0
$$

for a suitably chosen $q_{1}$. This also implies that $w_{1}$ is almost decreasing. Using [3, Lemma 5] (see also [3, Proposition 7] and [10, Proposition 2 (a)]) we get that $w_{1}$ is an essential weight. Similarly, since $v \in V_{m}$, we can find $q_{2}$ such that $w_{2}:=v / v_{q_{2}}$ is an essential weight. We now claim that ${ }^{\varepsilon} \Omega_{\varphi, \psi}^{v} \subseteq{ }^{\delta} \Omega_{\varphi, \psi}^{w}$ and that $1-\varepsilon \leqslant 1-\delta$ for some $\delta>0$.

Fix $z \in{ }^{\varepsilon} \Omega_{\varphi, \psi}^{v}$. Then, using the essentiality of $w$ (that is, $\widetilde{w} \leqslant c w$ ), followed by Schwarz' lemma applied on $w_{1}$ (that is, $w_{1}(\varphi(z)) \geqslant c_{1} w_{1}(z)$ ), we get that

$$
\begin{aligned}
\left|\tau_{\varphi, \psi}^{w}(z)\right| & \geqslant \frac{1}{c} \frac{|\psi(z)| v_{q_{1}}(z) w_{1}(\varphi(z))}{v_{q_{1}}(\varphi(z)) w_{1}(z)} \\
& \geqslant \frac{c_{1}}{c} \frac{|\psi(z)| v_{q_{1}}(z)}{v_{q_{1}}(\varphi(z))} .
\end{aligned}
$$

If $q_{1} \leqslant q_{2}$ we immediately get that

$$
\left|\tau_{\varphi, \psi}^{w}(z)\right| \geqslant \frac{c_{1}}{c} \frac{|\psi(z)| v_{q_{2}}(z)}{v_{q_{2}}(\varphi(z))} .
$$

On the other hand, if $q_{1}>q_{2}$ we let $n$ be the integer part of $q_{1} / q_{2}$ and proceed by noticing that

$$
\begin{aligned}
|\psi(z)|\left(\frac{1-|z|^{2}}{1-|\varphi(z)|^{2}}\right)^{q_{1}} & =|\psi(z)|\left(\frac{1-|z|^{2}}{1-|\varphi(z)|^{2}}\right)^{n q_{2}}\left(\frac{1-|z|^{2}}{1-|\varphi(z)|^{2}}\right)^{q_{1}-n q_{2}} \\
& \geqslant \frac{|\psi(z)|^{n+1}}{\|\psi\|_{\infty}^{n}}\left(\frac{1-|z|^{2}}{1-|\varphi(z)|^{2}}\right)^{(n+1) q_{2}}
\end{aligned}
$$


Hence,

$$
\left|\tau_{\varphi, \psi}^{w}(z)\right| \geqslant \frac{c_{1}}{c\|\psi\|_{\infty}^{n}}\left(\frac{|\psi(z)| v_{q_{2}}(z)}{v_{q_{2}}(\varphi(z))}\right)^{n+1} .
$$

As above, by using the fact that $w_{2}$ is almost decreasing combined with Schwarz' lemma (that is, $w_{2}(\varphi(z)) \geqslant c_{2} w_{2}(z)$ ), we get that

$$
\begin{aligned}
\frac{|\psi(z)| v_{q_{2}}(z)}{v_{q_{2}}(\varphi(z))} & =\frac{|\psi(z)| v(z) w_{2}(\varphi(z))}{v(\varphi(z)) w_{2}(z)} \\
& \geqslant c_{2} \frac{|\psi(z)| v(z)}{v(\varphi(z))} \\
& \geqslant c_{2} \varepsilon .
\end{aligned}
$$

In other words, by choosing

$$
\delta=\min \left\{\varepsilon, \frac{c_{1} c_{2} \varepsilon}{c}, \frac{c_{1}\left(c_{2} \varepsilon\right)^{n+1}}{\|\psi\|_{\infty}^{n}}\right\}
$$

we get that ${ }^{\varepsilon} \Omega_{\varphi, \psi}^{v} \subseteq{ }^{\delta} \Omega_{\varphi, \psi}^{w}$ and that $1-\varepsilon \leqslant 1-\delta$.

We shall now prove a Lipschitz condition for a function $f \in H_{v_{q}}^{\infty}$. A similar lemma can be found in [16] (Lemma 5.1), but since we obtain a better constant, we shall give a short proof for completeness.

LEMMA 3.6. For $f \in H_{v_{q}}^{\infty}$,

$$
\left|f(z)\left(1-|z|^{2}\right)^{q}-f(a)\left(1-|a|^{2}\right)^{q}\right| \leqslant R_{q}\|f\|_{v_{q}} \rho(z, a) \quad \text { for all } z, a \in \mathbb{D},
$$

where $R_{q}$ is the constant given by

$$
R_{q}:=\frac{1}{2}\left(2 q+\frac{(2(q+1))^{q+1}}{q^{q}}+\left(\left(2 q+\frac{(2(q+1))^{q+1}}{q^{q}}\right)^{2}+16\right)^{1 / 2}\right) .
$$

Proof: First we shall find an upper estimation for the total derivative of the function $h_{q}(z):=f(z)\left(1-|z|^{2}\right)^{q}$. A straightforward calculation gives that

$$
\left|h_{q}^{\prime}(z)\right| \leqslant\left|\frac{\partial h_{q}}{\partial z}\right|+\left|\frac{\partial h_{q}}{\partial \bar{z}}\right| \leqslant 2 q|f(z)|\left(1-|z|^{2}\right)^{q-1}+\left|f^{\prime}(z)\right|\left(1-|z|^{2}\right)^{q} .
$$

The Cauchy integral formula gives that for any $t>1$,

$$
f^{\prime}(z)=\frac{1}{2 \pi i} \int_{\Gamma_{1}} \frac{f(\xi)}{(\xi-z)^{2}} d \xi,
$$

where $\Gamma_{1}$ is the circle given by $|\xi-z|=\frac{1-|z|}{t}$. Thus,

$$
\begin{aligned}
\left|f^{\prime}(z)\right| & \leqslant \frac{1}{2 \pi} \frac{t^{2}}{(1-|z|)^{2}}\|f\|_{v_{q}} \int_{\Gamma_{1}} \frac{1}{(1-|\xi|)^{q}}|d \xi| \\
& \leqslant \frac{(2 t)^{q+1}}{(t-1)^{q}} \frac{\|f\|_{v_{q}}}{\left(1-|z|^{2}\right)^{q+1}},
\end{aligned}
$$


which is minimised when we choose $t=q+1$. Hence, we conclude that

$$
\left|h_{q}^{\prime}(z)\right| \leqslant\left(2 q+\frac{(2(q+1))^{q+1}}{q^{q}}\right) \frac{\|f\|_{v_{q}}}{\left(1-|z|^{2}\right)}=: K_{q} \frac{\|f\|_{v_{q}}}{\left(1-|z|^{2}\right)} .
$$

Next we shall find the desired estimate. Let $\Gamma_{2}$ be the geodesic in the hyperbolic geometry which connects the points $z \in \mathbb{D}$ and $a \in \mathbb{D}$. Then

$$
\begin{aligned}
\left|h_{q}(z)-h_{q}(a)\right| & \leqslant \int_{\Gamma_{2}}\left|h_{q}^{\prime}(\xi)\right||d \xi| \\
& \leqslant K_{q}\|f\|_{v_{q}} \int_{\Gamma_{2}} \frac{1}{\left(1-|\xi|^{2}\right)}|d \xi| \\
& =K_{q}\|f\|_{v_{q}} \beta(z, a) \\
& \leqslant K_{q}\|f\|_{v_{q}} \frac{\rho(z, a)}{1-\rho(z, a)^{2}},
\end{aligned}
$$

where the last inequality follows from the following calculation (using Taylor series expansion):

$$
\frac{1}{2} \log \frac{1+x}{1-x}=x+\frac{x^{3}}{3}+\frac{x^{5}}{5}+\cdots \leqslant x\left(1+x^{2}+x^{4}+\cdots\right)=\frac{x}{1-x^{2}} \quad(-1<x<1) .
$$

Let $s>1$. If $\rho(z, a)<s^{-1}$, then $1-\rho(z, a)^{2} \geqslant 1-s^{-2}$ and using the estimation above we get that

$$
\left|h_{q}(z)-h_{q}(a)\right| \leqslant \frac{s^{2}}{s^{2}-1} K_{q}\|f\|_{v_{q}} \rho(z, a) .
$$

On the other hand, if $\rho(z, a) \geqslant s^{-1}$ we immediately get that

$$
\left|h_{q}(z)-h_{q}(a)\right| \leqslant 2 s\|f\|_{v_{q}} \rho(z, a) .
$$

Solving the equation $s^{2} /\left(s^{2}-1\right) K_{q}=2 s$ for $s$ yields the desired constant.

THEOREM 3.7 .

(a) If $W_{\varphi, \psi}: H_{v_{q}}^{\infty} \rightarrow H_{v_{q}}^{\infty}$ has closed range, then there exists $\varepsilon>0$ such that

$$
\varphi\left({ }^{\varepsilon} \Omega_{\varphi, \psi}^{v_{q}}\right) \cap \overline{D(a, 1-\varepsilon)} \neq \emptyset \text { for all } a \in \mathbb{D} .
$$

(b) Let $R_{q}$ be the constant in Lemma 3.6. If there are $r \in\left(0, R_{q}^{-1}\right)$ and $\varepsilon>0$ such that

$$
\varphi\left({ }^{\varepsilon} \Omega_{\varphi, \psi}^{v_{q}}\right) \cap \overline{D(a, r)} \neq \emptyset \text { for all } a \in \mathbb{D}
$$

then $W_{\varphi, \psi}: H_{v_{q}}^{\infty} \rightarrow H_{v_{q}}^{\infty}$ has closed range.

Proof: (a) Assume that there exists $\delta>0$ such that $\left\|W_{\varphi, \psi} f\right\|_{v_{q}} \geqslant \delta\|f\|_{v_{q}}$ for all $f \in H_{v_{q}}^{\infty}$. Let $a \in \mathbb{D}$ and consider the function

$$
\sigma_{a}^{\prime}(z)^{q}=\left(\frac{1-|a|^{2}}{(1-\bar{a} z)^{2}}\right)^{q} .
$$


We have that $\left(\sigma_{a}^{\prime}\right)^{q} \in H_{v_{q}}^{\infty}$ and that $\left\|\left(\sigma_{a}^{\prime}\right)^{q}\right\|_{v_{q}}=1$. Indeed,

$$
\left\|\left(\sigma_{a}^{\prime}\right)^{q}\right\|_{v_{q}}=\sup _{z \in \mathbb{D}}\left|\sigma_{a}^{\prime}(z)\right|^{q}\left(1-|z|^{2}\right)^{q}=\sup _{z \in \mathbf{D}}\left(1-\left|\sigma_{a}(z)\right|^{2}\right)^{q}=1 .
$$

Thus, for any fixed $\varepsilon^{\prime}<\delta$ we can choose $z_{a} \in \mathbb{D}$ such that

$$
\left|\sigma_{a}^{\prime}\left(\varphi\left(z_{a}\right)\right)\right|^{q}\left|\psi\left(z_{a}\right)\right|\left(1-\left|z_{a}\right|^{2}\right)^{q} \geqslant \varepsilon^{\prime}
$$

and therefore

$$
\frac{\left|\psi\left(z_{a}\right)\right|\left(1-\left|z_{a}\right|^{2}\right)^{q}}{\left(1-\left|\varphi\left(z_{a}\right)\right|^{2}\right)^{q}}\left(1-\left|\sigma_{a}\left(\varphi\left(z_{a}\right)\right)\right|^{2}\right)^{q} \geqslant \varepsilon^{\prime}
$$

Hence, $z_{a} \in{ }^{\varepsilon^{\prime}} \Omega_{\varphi, \psi}^{v_{q}}$ and clearly

$$
\left(1-\left|\sigma_{a}\left(\varphi\left(z_{a}\right)\right)\right|^{2}\right)^{q} \geqslant \frac{\varepsilon^{\prime}}{T_{\varphi, \psi}^{v_{q}}} \text { gives }\left|\sigma_{a}\left(\varphi\left(z_{a}\right)\right)\right| \leqslant\left(1-\left(\frac{\varepsilon^{\prime}}{T_{\varphi, \psi}^{v_{q}}}\right)^{1 / q}\right)^{1 / 2} .
$$

By choosing $\varepsilon<\varepsilon^{\prime}$ small enough, the calculations above implies that $z_{a} \in{ }^{\varepsilon} \Omega_{\varphi, \psi}^{v_{q}}$ and that $\varphi\left(z_{a}\right) \in \overline{D(a, 1-\varepsilon)}$.

(b) We begin by observing that the assumption that $\varphi\left({ }^{\varepsilon} \Omega_{\varphi, \psi}^{v_{\varphi}}\right) \cap \overline{D(a, r)} \neq \emptyset$ for all $a \in \mathbb{D}$ is equivalent to the assumption that for all $a \in \mathbb{D}$ there exists $z_{a} \in{ }^{\varepsilon} \Omega_{\varphi, \psi}^{v_{q}}$ such that $\rho\left(\varphi\left(z_{a}\right), a\right) \leqslant r$. Take $f \in H_{v_{q}}^{\infty}$. Without loss of generality, we may assume that $\|f\|_{v_{q}}=1$. Thus, we can choose $a \in \mathbb{D}$ depending only on $f$ and $r$ such that

$$
|f(a)|\left(1-|a|^{2}\right)^{q} \geqslant \frac{1}{2}\left(1+r R_{q}\right) .
$$

Using Lemma 3.6 and the assumptions we get that

$$
\left|f(a)\left(1-|a|^{2}\right)^{q}-f\left(\varphi\left(z_{a}\right)\right)\left(1-\left|\varphi\left(z_{a}\right)\right|^{2}\right)^{q}\right| \leqslant r R_{q} .
$$

Therefore we conclude that

$$
\begin{aligned}
\left\|W_{\varphi, \psi} f\right\|_{v_{q}} & \geqslant\left|f\left(\varphi\left(z_{a}\right)\right)\right|\left|\psi\left(z_{a}\right)\right|\left(1-\left|z_{a}\right|^{2}\right)^{q} \\
& =\left|f\left(\varphi\left(z_{a}\right)\right)\right|\left(1-\left|\varphi\left(z_{a}\right)\right|^{2}\right)^{q}\left|\tau_{\varphi, \psi}^{v_{q}}\left(z_{a}\right)\right| \\
& \geqslant \varepsilon\left(|f(a)|\left(1-|a|^{2}\right)^{q}-r R_{q}\right) \\
& \geqslant \frac{\varepsilon}{2}\left(1-r R_{q}\right) .
\end{aligned}
$$

\section{ProOF OF THEOREM 3.2 .}

First we notice that we may assume that $\varphi(0)=0$ by Lemma 3.1 and that $W_{\varphi, \psi}$ is bounded on $H_{v}^{\infty}$. 
(a) As in the proof of Lemma 3.5, we can find $q$ such that $v / v_{q}$ is an essential weight. By Lemma 3.4 we know that $W_{\varphi, \psi}$ has closed range on $H_{v_{q}}^{\infty}$. Using Theorem 3.7 (a) we know that there exists $\varepsilon>0$ such that $\varphi\left({ }^{\varepsilon} \Omega_{\varphi, \psi}^{v_{\varphi}}\right) \cap \overline{D(a, 1-\varepsilon)} \neq \emptyset$ for all $a \in \mathbb{D}$. Lemma 3.5 finishes the proof.

(b) Clearly, with $q>\limsup _{|z| \rightarrow 1}\left(1-|z|^{2}\right)^{2}(-\Delta \log v(z))$, we get that

$$
-\Delta \log \frac{v_{q}(z)}{v(z)}=\frac{q}{\left(1-|z|^{2}\right)^{2}}-(-\Delta \log v(z))>0 \quad \text { near } \partial \mathbb{D} \text {. }
$$

That is, $v_{q} / v$ is an essential weight (see the proof of Lemma 3.5). Assume that for some $w \in V_{m}$ there are $r \in\left(0, R_{q}^{-1}\right)$ and $\varepsilon>0$ such that $\varphi\left({ }^{\varepsilon} \Omega_{\varphi, \psi}^{w}\right) \cap \overline{D(a, r)} \neq \emptyset$ for all $a \in \mathbb{D}$. Then Lemma 3.5 gives that there exists $\delta>0$ such that $\varphi\left({ }^{\delta} \Omega_{\varphi, \psi}^{v_{q}}\right) \cap \overline{D(a, r)} \neq \emptyset$ for all $a \in \mathbb{D}$. Theorem 3.7 (b) and Lemma 3.4 finish the proof.

COROLlary 3.8. Let $v \in V_{\mathrm{m}}$ and $\psi \in H^{\infty}$. Then $W_{\varphi, \psi}$ has closed range $H_{v}^{\infty}$ if and only if $M_{\psi}$ and $C_{\varphi}$ have closed range on $H_{v}^{\infty}$.

ProOF: By Lemma 3.1 we may assume that $\varphi(0)=0$. Clearly, if $M_{\psi}$ and $C_{\varphi}$ have closed range on $H_{v}^{\infty}$, then so does $W_{\varphi, \psi}$. Conversely, assume that there exists $\varepsilon>0$ such that $\left\|W_{\varphi, \psi} f\right\|_{v} \geqslant \varepsilon\|f\|_{v}$ for all $f \in H_{v}^{\infty}$. Take $f \in H_{v}^{\infty}$. Then

$$
\sup _{z \in \mathbb{D}}|f(\varphi(z))| v(z) \geqslant \sup _{z \in \mathbf{D}} \frac{|\psi(z)|}{\|\psi\|_{\infty}}|f(\varphi(z))| v(z) \geqslant \frac{\varepsilon}{\|\psi\|_{\infty}}\|f\|_{v} .
$$

That is, $C_{\varphi}$ has closed range on $H_{v}^{\infty}$. On the other hand, by using Theorem 3.2 (a), we know that there exists $\varepsilon>0$ such that

$$
\varphi\left({ }^{\varepsilon} \Omega_{\varphi, \psi}^{v_{1}}\right) \cap \overline{D(a, 1-\varepsilon)} \neq \emptyset \text { for all } a \in \mathbb{D} .
$$

Using Schwarz' lemma twice shows us that $\varphi\left({ }^{\varepsilon} \Omega_{\varphi, \psi}^{v_{1}}\right) \subseteq{ }^{\varepsilon} \Omega_{\varphi, \psi}^{v_{1}} \subseteq{ }^{\varepsilon} \Omega_{\psi}$. Thus,

$$
{ }^{\varepsilon} \Omega_{\psi} \cap \overline{D(a, 1-\varepsilon)} \neq \emptyset \text { for all } a \in \mathbb{D},
$$

which by [4, Lemma 3.7] mentioned earlier, implies that $M_{\psi}$ has closed range on $H_{v}^{\infty}$.

Corollary 3.9. (Non-moderate weights) Let $\varphi(0)=0$ and $\psi \in H^{\infty}$.

(a) (Necessity for rapidly decaying weights) Let $v \in C^{2}(\mathbb{D})$ be a weight which satisfies $\left(1-|z|^{2}\right)^{2}(-\Delta \log v(z)) \rightarrow \infty$ as $|z| \rightarrow 1$. If $W_{\varphi, \psi}$ has closed range on $H_{v}^{\infty}$, then for every $w \in V_{\mathrm{m}}$ there exists $\varepsilon>0$ such that

$$
\varphi\left({ }^{\varepsilon} \Omega_{\varphi, \psi}^{w}\right) \cap \overline{D(a, 1-\varepsilon)} \neq \emptyset \quad \text { for all } a \in \mathbb{D} .
$$

(b) (Sufficiency for slowly decaying weights) Let $R_{q}$ be as in Lemma 3.6. Let $v \in C^{2}(\mathbb{D})$ be a weight which satisfies $\left(1-|z|^{2}\right)^{2}(-\Delta \log v(z)) \rightarrow 0$ as $|z| \rightarrow 1$. If for any $q$ there are $r \in\left(0, R_{q}^{-1}\right)$ and $\varepsilon>0$ such that

$$
\varphi\left({ }^{\varepsilon} \Omega_{\varphi, \psi}^{v_{\varphi}}\right) \cap \overline{D(a, r)} \neq \emptyset \text { for all } a \in \mathbf{D} \text {, }
$$

then $W_{\varphi, \psi}$ has closed range on $H_{v}^{\infty}$. 
Proof: First we notice that $W_{\varphi, \psi}$ is bounded on $H_{v}^{\infty}$ for all almost decreasing weights.

(a) Notice that for any $q$ we have that $v / v_{q}$ is an essential weight. Indeed, for any $q$ there exists $r_{0}$ close enough to 1 such that

$$
-\Delta \log \frac{v(z)}{v_{q}(z)}=\frac{\left(1-|z|^{2}\right)^{2}(-\Delta \log v(z))-q}{\left(1-|z|^{2}\right)^{2}}>0 \text { for }|z| \geqslant r_{0} .
$$

Using Lemma 3.4 followed by Theorem 3.2 (a) finishes the proof.

(b) By a similar argumentation as above, we get that $v_{q} / v$ is an essential weight for any $q$. Hence, by using Theorem 3.7 (b) followed by Lemma 3.4 we get that $W_{\varphi, \psi}$ has closed range on $H_{v}^{\infty}$.

REMARK 3.10. In Corollary 3.9 (a) we are dealing with weights tending to zero very rapidly (usually weights satisfying condition $L_{2}$ but not $L_{1}$, like $v(z)=e^{-\left(1-|z|^{2}\right)^{-\gamma}}$, with $\gamma>0$ ). In Corollary 3.9 (b), on the other hand, we are dealing with weights either tending to zero very slowly or not at all (usually essential weights satisfying condition $L_{2}$ but not $L_{1}$, like $v(z)=(1-\log (1-|z|))^{-\gamma}$, with $\gamma>0$ or $\left.v(z) \equiv 1\right)$.

EXAMPLE 3.11. Assume that $v \in V_{m}$ (or that $v$ satisfies the conditions in Corollary $3.9(\mathrm{~b}))$. Let $\varphi(z)=z^{m}(m \in \mathbb{N})$ and let $\psi(z)=z^{n}(n \in \mathbb{N} \cup\{0\})$. Then $W_{\varphi, \psi}$ has closed range on $H_{v}^{\infty}$.

PROOF: Clearly the operator is bounded. We notice that for any $q$, we have that $\left|\tau_{\varphi, \psi}^{v_{q}}(z)\right|=0$ if and only if $z=0$. Moreover, $\lim _{|z| \rightarrow 1}\left|\tau_{\varphi, \psi}^{v_{q}}(z)\right|=m^{-q}$. In other words, by choosing $\varepsilon<\min \left\{m^{-q}, R_{q}^{-1 / m}\right\}$, we can make $\varphi\left({ }^{\varepsilon} \Omega_{\varphi, \psi}^{v_{q}}\right)$ cover the whole unit disk except for a disk with the origin as centre and radius less than $R_{q}^{-1}$. Using Theorem 3.2 (b) (or Corollary 3.9 (b)) finishes the proof.

For the case when $v \equiv 1$ we have that $C_{\varphi}$ has closed range on $H^{\infty}$ if and only if $\overline{\varphi(\mathbb{D})} \supseteq \partial \mathbb{D}$ (see [23, Theorem 3]). Similarly, $M_{\psi}: H^{\infty} \rightarrow H^{\infty}$ has closed range if and only if ess $\inf _{z \in \partial \mathbb{D}}\left|\psi^{*}(z)\right|>0$, where $\psi^{*}$ denotes the radial limit of $\psi$ (see [4, Proposition 3.2]). Note that $C_{\varphi}$ is always bounded on $H^{\infty}$, while $M_{\psi}$ (and hence $W_{\varphi, \psi}$ ) is bounded on $H^{\infty}$ if and only if $\psi \in H^{\infty}$. A complete characterisation of when $W_{\varphi, \psi}$ has closed range on $H^{\infty}$ in terms of Gelfand transforms and Shilov boundaries can be found in [21, Theorem 2.2].

Next we shall show that $\overline{\varphi(\mathbb{D})} \supseteq \partial \mathbb{D}$ is a necessary condition in a much more general setting than for the special case $v \equiv 1$ mentioned above.

COROLlary 3.12. (Weights of moderate or rapid decay) Let $\psi \in H^{\infty}$. Assume that $v \in V_{\mathrm{m}}$ (or that $\varphi(0)=0$ and that $v$ satisfies the conditions in Corollary $3.9(b))$. If $W_{\varphi, \psi}$ has closed range on $H_{v}^{\infty}$, then for every $w \in V_{\mathrm{m}}$ there exists $\varepsilon>0$ such that

$$
\overline{\varphi\left(\varepsilon \Omega_{\varphi, \psi}^{w}\right)} \supseteq \partial \mathbb{D} \text {. }
$$


PROOF: Suppose that for some $w \in V_{m}$, there exists a point $\eta \in \partial \mathbb{D}$ such that $\eta \notin \overline{\varphi\left({ }^{\varepsilon} \Omega_{\varphi, \psi}^{w}\right)}$ for any $\varepsilon>0$. Then for all $\varepsilon>0$ there exists a sequence $\left\{a_{n}\right\} \subseteq \mathbb{D} \backslash \varphi\left({ }^{\varepsilon} \Omega_{\varphi, \psi}^{w}\right)$, such that $a_{n} \rightarrow \eta$ as $n \rightarrow \infty$. Therefore,

$$
\lim _{n \rightarrow \infty}\left|\sigma_{a_{n}}(\varphi(z))\right|=\frac{|\eta-\varphi(z)|}{|1-\bar{\eta} \varphi(z)|}=1 \quad \text { for all } z \in{ }^{\varepsilon} \Omega_{\varphi, \psi}^{w} .
$$

Theorem 3.2 (a) (or Corollary 3.9 (b)) indicates that $W_{\varphi, \psi}$ cannot have closed range on $H_{v}^{\infty}$.

REMARK 3.13. If $v=v_{q}$ in Corollary 3.12, we could replace the assumption that $\psi \in H^{\infty}$ with the assumption that $W_{\varphi, \psi}$ is bounded on $H_{v_{q}}^{\infty}$ and still get that there exists $\varepsilon>0$ such that $\overline{\varphi^{\left(\varepsilon \Omega_{\varphi, \psi}^{\left.v_{q}\right)}\right.}} \supseteq \partial \mathbb{D}$. (see Theorem $3.7(\mathrm{a})$ ).

REMARK 3.14. Corollary 3.12 also reflects the intuitively evident fact that if $W_{\varphi, \psi}$ is compact on $H_{v}^{\infty}$ then it cannot have closed range on $H_{v}^{\infty}$. Indeed, there exists $\varepsilon>0$ such that $\overline{\varphi\left(\varepsilon \Omega_{\varphi, \psi}^{v}\right)} \supseteq \partial \mathbb{D}$ if and only if there exists $\varepsilon>0$ such that

$$
\text { for all } \eta \in \partial \mathbb{D}: \limsup _{\varphi(z) \rightarrow \eta}\left|\tau_{\varphi, \psi}^{v}(z)\right| \geqslant \varepsilon \text {. }
$$

Thus, using [8, Corollary 4.3], $W_{\varphi, \psi}$ cannot be compact if it has closed range. This can of course be seen in many other ways as well.

\section{ASSOCIATION WITH CONFORMAL MAPPINGS}

When restricted to the special case where $\varphi$ is univalent (that is, $\varphi^{\prime}(z) \neq 0$ for all $z \in \mathbb{D})$ and $\psi=\left(\varphi^{\prime}\right)^{\mu}$, we get a complete characterisation of when $W_{\varphi,\left(\varphi^{\prime}\right)^{\mu}}$ has closed range on $H_{v_{q}}^{\infty}$ for $q \geqslant \mu$ (the Schwarz-Pick lemma guarantees that $W_{\varphi,\left(\varphi^{\prime}\right) \mu}$ is bounded). Under the additional hypothesis that $\varphi^{\prime} \in H^{\infty}$, we obtain a more general characterisation. Some of the techniques we use are inspired by the ones used in [14].

TheORem 4.1. Let $\varphi$ be univalent and fix $\mu$. Assume that $q \geqslant \mu$. Then $W_{\varphi,\left(\varphi^{\prime}\right)^{\mu}}$ has closed range on $H_{v_{q}}^{\infty}$ if and only if there exists $\varepsilon>0$ such that

$$
\varphi\left({ }^{\varepsilon} \Omega_{\varphi,\left(\varphi^{\prime}\right) \mu}^{\left.v_{\varphi}\right)}\right) \overline{D(a, 1-\varepsilon)} \neq \emptyset \quad \text { for all } a \in \mathbb{D} \text {. }
$$

To be able to prove Theorem 4.1 we need the following three lemmas.

LEMMA 4.2. For $p>1$,

$$
\|f\|_{v_{q}} \approx \sup _{a \in \mathbf{D}}\left(\int_{\mathbf{D}}|f(z)|^{2 / q}\left(1-\left|\sigma_{a}(z)\right|^{2}\right)^{p} d A(z)\right)^{q / 2}
$$

Proof: Assume that $p>1$. Then

$$
\begin{aligned}
\sup _{a \in \mathbf{D}}\left(\int_{\mathbf{D}}|f(z)|^{2 / q}(1\right. & \left.\left.-\left|\sigma_{a}(z)\right|^{2}\right)^{p} d A(z)\right)^{q / 2} \\
& \leqslant\|f\|_{v_{q}}\left(\sup _{a \in \mathbf{D}}\left(1-|a|^{2}\right)^{p} \int_{\mathbf{D}} \frac{\left(1-|z|^{2}\right)^{p-2}}{|1-\bar{a} z|^{2 p}} d A(z)\right)^{q / 2} \\
& \leqslant c_{p}\|f\|_{v_{q}},
\end{aligned}
$$


where the last inequality follows from the integral formula (see [28, Lemma 4.2.2]), which states that for $a \in \mathbb{D}, c \in \mathbb{R}$ and $t>-1$,

$$
\int_{\mathbb{D}} \frac{\left(1-|z|^{2}\right)^{t}}{|1-\bar{a} z|^{2+t+c}} d A(z) \approx \begin{cases}\text { bounded in } a & \text { for } c<0 \\ \log \left(1-|a|^{2}\right)^{-1} & \text { for } c=0 \\ \left(1-|a|^{2}\right)^{-c} & \text { for } c>0\end{cases}
$$

Conversely, by a change of $z \mapsto \sigma_{a}(z)$ we obtain

$$
\begin{aligned}
\sup _{a \in \mathbb{D}}\left(\int_{\mathbb{D}}|f(z)|^{2 / q}\right. & \left.\left(1-\left|\sigma_{a}(z)\right|^{2}\right)^{p} d A(z)\right)^{q / 2} \\
& =\sup _{a \in \mathbb{D}}\left(\int_{\mathbb{D}}\left|f\left(\sigma_{a}(z)\right)\right|^{2 / q}\left(1-|z|^{2}\right)^{p} \frac{\left(1-|a|^{2}\right)^{2}}{|1-\bar{a} z|^{4}} d A(z)\right)^{q / 2} \\
& \geqslant \sup _{a \in \mathbb{D}}\left(1-|a|^{2}\right)^{q}\left(2 \int_{0}^{1} \frac{1}{2 \pi} \int_{0}^{2 \pi}\left|f\left(\sigma_{a}\left(r e^{i \theta}\right)\right)\right|^{2 / q} d \theta\left(1-r^{2}\right)^{p} r d r\right)^{q / 2} \\
& \geqslant \sup _{a \in \mathbb{D}}|f(a)|\left(1-|a|^{2}\right)^{q}\left(\int_{0}^{1} 2 r\left(1-r^{2}\right)^{p} d r\right)^{q / 2} \\
& =\left(\frac{1}{p+1}\right)^{q / 2}\|f\|_{v_{q}},
\end{aligned}
$$

where the last inequality follows from the subharmonicity of $\left|f \circ \sigma_{a}\right|^{2 / a}$.

REMARK 4.3. We have that $H_{v_{1}}^{\infty}=N_{p}$ for $p>1$. That is, the $N_{p}$-spaces, of which $N_{1}$ was introduced in [17] and where

$$
N_{p}:=\left\{f \in H(\mathbb{D}): \sup _{a \in \mathbb{D}}\left(\int_{\mathbb{D}}|f(z)|^{2}\left(1-\left|\sigma_{a}(z)\right|^{2}\right)^{p} d A(z)\right)^{1 / 2}<\infty\right\} .
$$

LEMMA 4.4. If there exists $\varepsilon>0$ such that the set $\varphi\left({ }^{\varepsilon} \Omega_{\varphi_{(}\left(\varphi^{\prime}\right)^{\mu}}^{v_{q}}\right)$ satisfies the reverse Carleson condition, then $W_{\varphi,\left(\varphi^{\prime}\right) \mu}$ has closed range on $H_{v_{q}}^{\infty}$.

Proof: Luecking [18] proved that a set $K \subseteq \mathbb{D}$ satisfies the reverse Carleson condition if and only if there exists $k>1$ such that

$$
\int_{\mathbb{D}}|f(z)|^{p}\left(1-|z|^{2}\right)^{q} d A(z) \leqslant k \int_{K}|f(z)|^{p}\left(1-|z|^{2}\right)^{q} d A(z) \quad \text { for all } f \in A_{q}^{p}
$$

where $0<p<\infty$ and where $A_{q}^{p}$ is the weighted Bergman space (with norm $\|\cdot\|_{A_{q}^{p}}$ ) given by

$$
A_{q}^{p}:=\left\{f \in H(\mathbb{D}):\|f\|_{A_{q}^{p}}=\left(\int_{\mathbb{D}}|f(z)|^{p}\left(1-|z|^{2}\right)^{q} d A(z)\right)^{1 / p}<\infty\right\} .
$$

See Main Theorem on p. 2, condition ( $\left.2^{\prime \prime}\right)$ on p. 5 and the remarks on p. 10 in [18] for more details (note that due to a typing error the $p$ 's are missing in the remarks on p. 10). Take $f \in H_{v_{q}}^{\infty}$. Then, using Lemma 4.2 (with $p=2$ ) we get that

$$
\|f\|_{v_{q}} \approx \sup _{a \in \mathbb{D}}\left(\int_{\mathbb{D}}\left|f(z) \cdot \sigma_{a}^{\prime}(z)^{q}\right|^{2 / q}\left(1-|z|^{2}\right)^{2} d A(z)\right)^{q / 2} \text {. }
$$


Thus, $f(z)\left(\sigma_{a}^{\prime}\right)^{q} \in A_{2}^{2 / q}$ and we can use the reverse Carleson condition to get that

$$
\begin{aligned}
& \|f\|_{v_{q}} \leqslant 3^{q / 2} k \sup _{a \in \mathbb{D}}\left(\int_{\varphi\left({ }^{(\varepsilon} \Omega_{\varphi,\left(\varphi^{\prime}\right) \mu}^{\left.v_{q}\right)}\right.}|f(z)|^{2 / q}\left(1-\left|\sigma_{a}(z)\right|^{2}\right)^{2} d A(z)\right)^{q / 2} \\
& \leqslant 3^{q / 2} k c_{p} \sup _{z \in \varphi\left(c \Omega^{\left.v_{\varphi},\left(\varphi^{\prime}\right)^{\mu}\right)}\right.}|f(z)|\left(1-|z|^{2}\right)^{q} \\
& =3^{q / 2} k c_{p} \sup _{z \in^{\in} \Omega_{\varphi,\left(\varphi^{\prime}\right)^{\mu}}^{v_{q}}}|f(\varphi(z))|\left|\varphi^{\prime}(z)\right|^{\mu}\left(1-|z|^{2}\right)^{q} \frac{\left(1-|\varphi(z)|^{2}\right)^{q}}{\left|\varphi^{\prime}(z)\right|^{\mu}\left(1-|z|^{2}\right)^{q}} \\
& \leqslant \frac{3^{q / 2} k c_{p}}{\varepsilon}\left\|W_{\varphi,\left(\varphi^{\prime}\right) \mu} f\right\|_{v_{q}}
\end{aligned}
$$

where the second inequality follows from the integral formula which we also used in the proof of Lemma 4.2.

LEMMA 4.5. The function $\tau_{\varphi,\left(\varphi^{\prime}\right)^{\mu}}^{v_{q}}$ is Lipschitz, or more precisely,

$$
\left|\tau_{\varphi,\left(\varphi^{\prime}\right)^{\mu}}^{v_{q}}(z)-\tau_{\varphi,\left(\varphi^{\prime}\right)^{\mu}}^{v_{q}}(a)\right| \leqslant R_{q, \mu} T_{\varphi,\left(\varphi^{\prime}\right)^{\mu}}^{v_{q}} \rho(z, a) \quad \text { for all } z, a \in \mathbb{D}
$$

where $R_{q, \mu}:=\left(2 q+3 \mu+\left((2 q+3 \mu)^{2}+4\right)^{1 / 2}\right) / 2$.

Proof: As in Lemma 3.6, we begin by finding an upper estimate for the total derivative of the function $\tau_{\varphi,\left(\varphi^{\prime}\right) \mu}^{v_{q}}$. Straightforward calculations give that

$$
\frac{\partial \tau_{\varphi,\left(\varphi^{\prime}\right)^{\mu}}^{v_{q}}}{\partial z}=\frac{\left(1-|z|^{2}\right)^{q} \varphi^{\prime}(z)^{\mu}}{\left(1-|\varphi(z)|^{2}\right)^{q}}\left(\frac{-\bar{z} q}{1-|z|^{2}}+\frac{\mu \varphi^{\prime \prime}(z)}{\varphi^{\prime}(z)}+\frac{q \overline{\varphi(z)} \varphi^{\prime}(z)}{1-|\varphi(z)|^{2}}\right)
$$

and

$$
\frac{\partial \tau_{\varphi,\left(\varphi^{\prime}\right)^{\mu}}^{v^{v^{\prime}}}}{\partial \bar{z}}=\frac{\left(1-|z|^{2}\right)^{q} \varphi^{\prime}(z)^{\mu}}{\left(1-|\varphi(z)|^{2}\right)^{q}}\left(\frac{-z q}{1-|z|^{2}}+\frac{q \varphi(z) \overline{\varphi^{\prime}(z)}}{1-|\varphi(z)|^{2}}\right) .
$$

The Schwarz-Pick lemma and a consequence of the verification of the Bieberbach conjecture for the second coefficient (see [22, Proposition 1.2]) give that

$$
\frac{\left|\varphi^{\prime}(z)\right|}{1-|\varphi(z)|^{2}} \leqslant \frac{1}{1-|z|^{2}} \text { and } \frac{\left|\varphi^{\prime \prime}(z)\right|}{\left|\varphi^{\prime}(z)\right|} \leqslant \frac{6}{1-|z|^{2}}
$$

respectively. Thus,

$$
\left|\left(\tau_{\varphi_{1}\left(\varphi^{\prime}\right)^{\mu}}^{v_{q}}\right)^{\prime}(z)\right| \leqslant 2(2 q+3 \mu) T_{\varphi_{,}\left(\varphi^{\prime}\right)^{\mu}}^{v_{v_{q}}} \frac{1}{1-|z|^{2}} .
$$

Continuing as in Lemma 3.6, we get that for $s>1$,

$$
\left|\tau_{\varphi,\left(\varphi^{\prime}\right)^{\mu}}^{v_{q}}(z)-\tau_{\varphi,\left(\varphi^{\prime}\right)^{\mu}}^{v_{q}}(a)\right| \leqslant \max \left\{2 s, \frac{2 s^{2}}{s^{2}-1}(2 q+3 \mu)\right\} T_{\varphi,\left(\varphi^{\prime}\right)^{\mu}}^{v_{q}} \rho(z, a) .
$$

Solving the equation $s^{2} /\left(s^{2}-1\right)(2 q+3 \mu)=2 s$ for $s$ finishes the proof. 


\section{ProOF OF THEOREM 4.1.}

Since the "only if" direction is an immediate consequence of Theorem 3.7 (a) it suffices to show the "if" direction. Hence, we assume that there exists $\delta>0$ such that $\varphi\left({ }^{\delta} \Omega_{\varphi_{\left(, \varphi^{\prime}\right)}}^{v_{\varphi}}\right) \cap \overline{D(a, 1-\delta)} \neq \emptyset$ for all $a \in \mathbb{D}$. We shall show that this implies that there

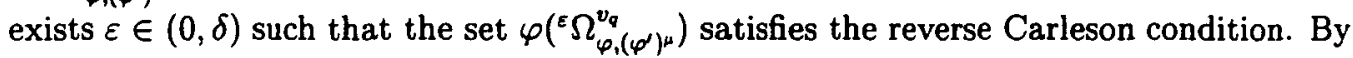
Lemma 4.4 we then know that $W_{\varphi,\left(\varphi^{\prime}\right)^{4}}$ has closed range on $H_{v_{q}}^{\infty}$. We shall divide the proof into two parts and show that there are constants $t \in(0,1)$ and $c \in(0,1)$ such that for all $a \in \mathbb{D}$ there exists $z_{a} \in \mathbb{D}$ and $s \in(0,1)$ such that

$$
\left|D(a, t) \cap \varphi\left({ }^{\varepsilon} \Omega_{\varphi,\left(\varphi^{\prime}\right)^{\mu}}^{v_{q}}\right)\right| \geqslant\left|\varphi\left(D\left(z_{a}, s\right)\right)\right| \stackrel{i i)}{\geqslant} c|D(a, t)| .
$$

For our convenience we shall denote $1-\delta$ by $r_{0}$ throughout the rest of the proof.

(i) For any $a \in \mathbb{D}$ we can choose $z_{a} \in{ }^{\delta} \Omega_{\varphi,\left(\varphi^{\prime}\right) \mu}^{v_{q}}$ such that $\rho\left(a, \varphi\left(z_{a}\right)\right) \leqslant r_{0}$. Let $R_{q, \mu}$ be the constant in Lemma 4.5 and fix $\varepsilon \in(0, \delta)$. Then put

$$
s=\frac{\delta-\varepsilon}{R_{q, \mu} T_{\varphi,\left(\varphi^{\prime}\right)^{\mu}}^{v_{v^{\prime}}}}
$$

and let $\lambda \in D\left(z_{a}, s\right)$, so that $\rho\left(z_{a}, \lambda\right)<s$. By using Lemma 4.5 we get that

$$
\left|\tau_{\varphi,\left(\varphi^{\prime}\right)^{\mu}}^{v_{q}}\left(z_{a}\right)-\tau_{\varphi,\left(\varphi^{\prime}\right)^{\mu}}^{v_{q}}(\lambda)\right|<\delta-\varepsilon .
$$

Thus, $\left|\tau_{\varphi,\left(\varphi^{\prime}\right)^{\mu}}^{v_{q}}(\lambda)\right|>\varepsilon$, which implies that $\varphi(\lambda) \in \varphi\left({ }^{\varepsilon} \Omega_{\varphi,\left(\varphi^{\prime}\right)^{\mu}}^{v_{q}}\right)$. Hence, we get that $\varphi\left(D\left(z_{a}, s\right)\right) \subseteq \varphi\left({ }^{\varepsilon} \Omega_{\varphi,\left(\varphi^{\prime}\right)}^{v_{q}}\right)$.

On the other hand,

$$
\begin{aligned}
\rho(a, \varphi(\lambda)) & \leqslant \frac{\rho\left(a, \varphi\left(z_{a}\right)\right)+\rho\left(\varphi\left(z_{a}\right), \varphi(\lambda)\right)}{1+\rho\left(a, \varphi\left(z_{a}\right)\right) \rho\left(\varphi\left(z_{a}\right), \varphi(\lambda)\right)} \\
& <\frac{r_{0}+s}{1+r_{0} s},
\end{aligned}
$$

where the last inequality follows from the fact that for every $x \leqslant r_{0}$ and $y<s$ we have that $(x+y) /(1+x y)<\left(r_{0}+s\right) /\left(1+r_{0} s\right)$. Let $t=\left(r_{0}+s\right) /\left(1+r_{0} s\right)$. Then $\varphi(\lambda) \in D(a, t)$, which in other words implies that $\varphi\left(D\left(z_{a}, s\right)\right) \subseteq D(a, t)$. Clearly, the above calculations prove the given inequality.

(ii) According to Lemma 3.1 we may assume that $\varphi(0)=0$. Using the univalence of $\varphi$ we get that

$$
\begin{aligned}
\left|\varphi\left(D\left(z_{a}, s\right)\right)\right| & =\int_{D\left(z_{a}, s\right)}\left|\varphi^{\prime}(\lambda)\right|^{2} d A(\lambda) \\
& =\int_{D\left(z_{a}, s\right)}\left(\frac{\left|\varphi^{\prime}(\lambda)\right|^{\mu}\left(1-|\lambda|^{2}\right)^{q}}{\left(1-|\varphi(\lambda)|^{2}\right)^{q}}\right)^{2 / \mu}\left(\frac{1-|\varphi(\lambda)|^{2}}{1-|\lambda|^{2}}\right)^{(2 q) / \mu} d A(\lambda) \\
& \geqslant(\varepsilon)^{2 / \mu} \int_{D\left(z_{a}, s\right)} \frac{\left(1-|\varphi(\lambda)|^{2}\right)^{2}}{\left(1-|\lambda|^{2}\right)^{2}} d A(\lambda),
\end{aligned}
$$


where we have used Schwarz' lemma and the proof of (i) in the inequality. A straightforward calculation gives that for $\lambda \in D\left(z_{a}, s\right)$,

$$
\frac{1-s^{2}}{4}\left(1-\left|z_{a}\right|^{2}\right)<1-|\lambda|^{2}<\frac{4}{1-s^{2}}\left(1-\left|z_{a}\right|^{2}\right)
$$

and by the Schwarz-Pick lemma we get that $\varphi(\lambda) \in D\left(\varphi\left(z_{a}\right), s\right)$. Therefore,

$$
\begin{aligned}
\left|\varphi\left(D\left(z_{a}, s\right)\right)\right| & \geqslant(\varepsilon)^{2 / \mu} \frac{\left(1-s^{2}\right)^{4}}{256} \frac{\left(1-\left|\varphi\left(z_{a}\right)\right|^{2}\right)^{2}}{\left(1-\left|z_{a}\right|^{2}\right)^{2}} \int_{D\left(z_{a}, s\right)} d A(\lambda) \\
& \geqslant(\varepsilon)^{2 / \mu} \frac{\left(1-r_{0}^{2}\right)^{2}\left(1-s^{2}\right)^{4}}{4096} \frac{\left(1-|a|^{2}\right)^{2}}{\left(1-\left|z_{a}\right|^{2}\right)^{2}}\left|D\left(z_{a}, s\right)\right|,
\end{aligned}
$$

where we have used the fact that $\rho\left(a, \varphi\left(z_{a}\right)\right) \leqslant r_{0}$ in the last inequality. Furthermore, one can easily check that

$$
s^{2}\left(1-\left|z_{a}\right|^{2}\right)^{2} \leqslant\left|D\left(z_{a}, s\right)\right| \leqslant \frac{s^{2}}{\left(1-s^{2}\right)^{2}}\left(1-\left|z_{a}\right|^{2}\right)^{2} .
$$

Hence,

$$
\begin{aligned}
\left|\varphi\left(D\left(z_{a}, s\right)\right)\right| & \geqslant(\varepsilon)^{2 / \mu} \frac{\left(1-r_{0}^{2}\right)^{2} s^{2}\left(1-s^{2}\right)^{4}}{4096} \frac{\left(1-t^{2}\right)^{2}}{t^{2}}|D(a, t)| \\
& =\left(\frac{\left(1-r_{0}^{2}\right)^{2} s\left(1-s^{2}\right)^{3}}{64\left(r_{0}+s\right)\left(1+r_{0} s\right)}(\varepsilon)^{1 / \mu}\right)^{2}|D(a, t)| .
\end{aligned}
$$

Corollary 4.6. Let $\varphi$ be univalent and assume that $\varphi^{\prime} \in H^{\infty}$. Then $W_{\varphi,\left(\varphi^{\prime}\right)^{\mu}}$ has closed range on $H_{v_{q}}^{\infty}$ (for any $\mu$ and any $q$ ) if and only if there exists $\varepsilon>0$ such that

$$
\varphi\left({ }^{\varepsilon} \Omega_{\varphi, \phi^{\prime}}^{v_{1}}\right) \cap \overline{D(a, 1-\varepsilon)} \neq \emptyset \text { for all } a \in \mathbb{D}
$$

ProOF: By Lemma 3.1 we may assume that $\varphi(0)=0$ and since $\varphi^{\prime} \in H^{\infty}$, we have

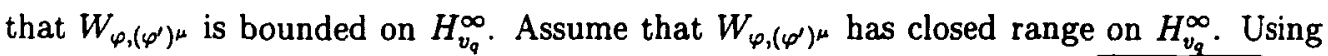
Theorem 3.2 (a) we know that there exists $\delta>0$ such that $\varphi\left({ }^{\delta} \Omega_{\varphi,\left(\varphi^{\prime}\right)^{\mu}}^{v_{\mu}}\right) \cap \overline{D(a, 1-\delta)} \neq \emptyset$ for all $a \in \mathbb{D}$. By letting $\varepsilon:=\min \left\{\delta, \delta^{1 / \mu}\right\}$, it is easy to see that $\varphi\left({ }^{\varepsilon} \Omega_{\varphi, \varphi^{\prime}}^{v_{1}}\right) \cap \overline{D(a, 1-\varepsilon)} \neq \emptyset$ for all $a \in \mathbb{D}$.

Conversely, assume that there exists $\varepsilon>0$ such that $\varphi\left({ }^{\varepsilon} \Omega_{\varphi, \varphi^{\prime}}^{v_{1}}\right) \cap \overline{D(a, 1-\varepsilon)} \neq \emptyset$ for all $a \in \mathbb{D}$. We shall show that if there exists $\varepsilon>0$ such that the set $\varphi\left({ }^{E} \Omega_{\varphi, \varphi^{\prime}}^{v_{1}}\right)$ satisfies the reverse Carleson condition, then $W_{\varphi,\left(\varphi^{\prime}\right)^{\mu}}$ has closed range on $H_{v_{q}}^{\infty}$ (compare this to Lemma 4.4). We can then apply the corresponding part of the proof of Theorem 4.1 (with $q=\mu=1$ ) to finish the proof. The only change in the proof of Lemma 4.4 that we have to make (except for the obvious ones) is in the last inequality. If $q \geqslant \mu$ we get

$$
\frac{\left(1-|\varphi(z)|^{2}\right)^{q}}{\left|\varphi^{\prime}(z)\right|^{\mu}\left(1-|z|^{2}\right)^{q}}=\left(\frac{1-|\varphi(z)|^{2}}{\left|\varphi^{\prime}(z)\right|\left(1-|z|^{2}\right)}\right)^{q}\left|\varphi^{\prime}(z)\right|^{q-\mu} \leqslant \frac{\left\|\varphi^{\prime}\right\|_{\infty}^{q-\mu}}{\varepsilon^{q}}
$$


while if $q<\mu$ we get (using Schwarz' lemma)

$$
\frac{\left(1-|\varphi(z)|^{2}\right)^{q}}{\left|\varphi^{\prime}(z)\right|^{\mu}\left(1-|z|^{2}\right)^{q}}=\left(\frac{1-|\varphi(z)|^{2}}{\left|\varphi^{\prime}(z)\right|\left(1-|z|^{2}\right)}\right)^{\mu}\left(\frac{1-|z|^{2}}{1-|\varphi(z)|^{2}}\right)^{\mu-q} \leqslant \frac{1}{\varepsilon^{\mu}} \text {. }
$$

Corollary 4.7. (Moderate weights) Assume that $\varphi$ is univalent and that $\varphi^{\prime} \in H^{\infty}$. Then $W_{\varphi,\left(\varphi^{\prime}\right)^{\mu}}$ has closed range on $H_{v}^{\infty}$ (for any $\mu$ and any $v \in V_{\mathrm{m}}$ ) if and only if there exists $\varepsilon>0$ such that

$$
\varphi\left({ }^{\varepsilon} \Omega_{\varphi, \varphi^{\prime}}^{v_{1}}\right) \cap \overline{D(a, 1-\varepsilon)} \neq \emptyset \text { for all } a \in \mathbb{D} \text {. }
$$

Proof: As in the proof of Corollary 3.8 we begin by noticing that we may assume that $\varphi(0)=0$ and that $W_{\varphi,\left(\varphi^{\prime}\right)^{\mu}}$ is bounded on $H_{v}^{\infty}$. Assume that $W_{\varphi,\left(\varphi^{\prime}\right)^{\mu}}$ has closed range on $H_{v}^{\infty}$. By choosing $q_{2}<\lim \sup \left(1-|z|^{2}\right)^{2}(-\Delta \log v(z))$ we easily see that $v / v_{q_{2}}$ is an essential weight (see the proof of Lemma 3.5). Using Lemma 3.4 followed by Corollary 4.6 we get the desired condition.

Conversely, assume that there exists $\varepsilon>0$ such that $\varphi\left({ }^{\varepsilon} \Omega_{\varphi, \varphi^{\prime}}^{\nu_{1}}\right) \cap \overline{D(a, 1-\varepsilon)} \neq \emptyset$ for all $a \in \mathbb{D}$. Choose $q_{1}>\limsup \left(1-|z|^{2}\right)^{2}(-\Delta \log v(z))$, so that $v_{q_{1}} / v$ becomes an essential weight. Using Corollary 4.6 we get that $W_{\varphi,\left(\varphi^{\prime}\right)^{\mu}}$ has closed range on $H_{v_{q_{1}}}^{\infty}$. Lemma 3.4 finishes the proof.

REMARK 4.8. The assumption that $\varphi^{\prime} \in H^{\infty}$ is kind of restrictive and makes Corollary 4.6 and Corollary 4.7 loose some of their significance. Nevertheless, they can be applied to for example automorphisms of the unit disk (see Example 4.9).

ExAmple 4.9. Fix $a \in \mathbb{D}, \gamma \in \mathbb{R}$ and let $v(z)=\left(1-|z|^{2}\right)^{q}\left(1-\log \left(1-|z|^{2}\right)\right)^{-\gamma}$. Then $W_{\sigma_{a},\left(\sigma_{a}^{\prime}\right)^{\mu}}$ has closed range on $H_{v}^{\infty}$ for any $\mu$.

PROOF: Straightforward calculations give that $v \in V_{m}$ and that $\sigma_{a}^{\prime} \in H^{\infty}$. Since $\left|\tau_{\sigma_{a}, \sigma_{a}^{\prime}}^{v_{1}}(z)\right|=1$ for all $z \in \mathbb{D}$, we get that ${ }^{\varepsilon} \Omega_{\sigma_{a}, \sigma_{a}^{\prime}}^{v_{1}}=\mathbb{D}$ for any $0<\varepsilon<1$. Corollary 4.7 finishes the proof.

\section{EXAMPLES}

In this section we take a more concrete look at the closed range property by giving some enlightening examples. That is, examples where $\overline{\varphi(\mathbb{D})} \supseteq \partial \mathbb{D}$, but $\overline{\varphi(\mathbb{D})} \neq \overline{\mathbb{D}}$ or $\varphi(\mathbb{D}) \neq \mathbb{D}$. Producing such analytic functions in an explicit form is very difficult, even numerically, except in some simple cases (like when the omitted region produced by $\varphi$ is a disk as in Example 5.1). The original Schwarz-Christoffel mapping cannot be used since it only provides mappings from the unit disk to the interior of a polygon. However, by letting the sides in the polygon be circular arcs, such functions could be found in theory. Two attempts to implement this numerically have been made (see $[6,15])$ but the techniques are still quite experimental (see [11] for more on this topic). 
The next example will show that if the omitted region produced by $\varphi$ is a disk which is tangent to the boundary of the unit disk, then $W_{\varphi, \psi}$ cannot have closed range on $H_{v}^{\infty}$ $\left(v \in V_{m}\right.$ and $\left.\psi \in H^{\infty}\right)$. Nevertheless, it might have closed range on $H^{\infty}$.

EXAMPLE 5.1. Let $v \in V_{m}$ and $\psi \in H^{\infty}$. Assume that

$$
\underset{z \in a \mathbb{D}}{\operatorname{essinf}}\left|\psi^{*}(z)\right|>0 \quad \text { and } \quad \varphi(z)=\frac{\log ((1+z) /(1-z))}{\pi i+\log ((1+z) /(1-z))} \quad\left(=\frac{2 \operatorname{arctanh}(z)}{\pi i+2 \operatorname{arctanh}(z)}\right) \text {. }
$$

Then $W_{\varphi, \psi}$ has closed range on $H^{\infty}$, but not on $H_{v}^{\infty}$ (see Figure 1).
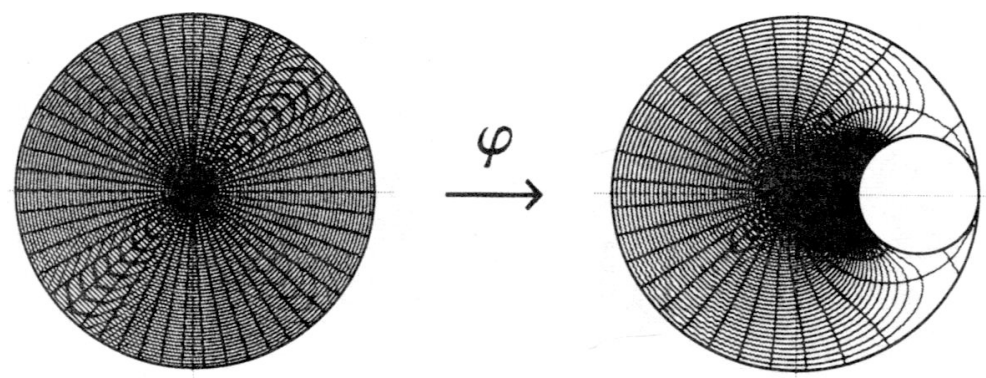

Figure 1: A density plot of $\varphi$ in Example 5.1.

PROOF: Clearly $W_{\varphi, \psi}$ is bounded on both $H^{\infty}$ and $H_{v}^{\infty}$. By the results in $[23,4]$, mentioned before Corollary 3.12 , we know that $W_{\varphi, \psi}$ has closed range on $H^{\infty}$. On the other hand, we notice that for any given radius $r$, we have that $\lim _{a \rightarrow 1}|\overline{D(a, r)}|=0$. Therefore it is easy to see that for all $\varepsilon>0$ we can always choose $a$ real and close enough to 1 , so that $\varphi(\mathbb{D}) \cap \overline{D(a, 1-\varepsilon)}=\emptyset$. Theorem 3.2 (a) gives that $W_{\varphi, \psi}$ cannot have closed range on $H_{v}^{\infty}$.

Example 5.1 might lead one to believe that $\overline{\varphi(\mathbb{D})}=\overline{\mathbb{D}}$ is a necessary condition for the closed range property. However, as the two next examples will show, $\overline{\varphi(\mathbb{D})}=\overline{\mathbb{D}}$ is neither a necessary nor a sufficient condition (generally speaking) for a weighted composition operator to have closed range on $H_{v}^{\infty}$. Not even when $\varphi$ is univalent. The first example is an improved version of [14, Example 2], while the second one uses an idea of [25, Example 6.5].

EXAMPLE 5.2. Fix $q$ and $r_{0} \in\left(0, R_{q}^{-1}\right)$, where $R_{q}$ is the constant given in Lemma 3.6. Let $G=\mathbb{D} \backslash\left\{\overline{D\left(0, r_{0}\right)} \cup\left(r_{0}, 1\right)\right\}$ and let $\varphi$ be the univalent mapping that maps $\mathbb{D}$ onto

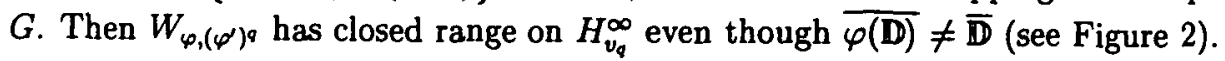

Proof: First we notice that $W_{\varphi,\left(\varphi^{\prime}\right)}$ is bounded on $H_{v_{q}}^{\infty}$ due to the Schwarz-Pick lemma. In order to use Theorem 3.7 (b), we need to study the behaviour of the function $\tau_{\varphi,\left(\varphi^{\prime}\right) \text { a }}^{v_{q}}$. Using Koebe's one-quarter theorem (see [22, Corollary 1.4]), it follows that

$$
\frac{1}{2^{q}}\left(\frac{\operatorname{dist}(\varphi(z), \partial \varphi(\mathbf{D}))}{\operatorname{dist}(\varphi(z), \partial \mathbf{D})}\right)^{q} \leqslant\left|\tau_{\varphi,\left(\varphi^{\prime q}\right)}^{v_{q}}(z)\right| \leqslant 4^{q}\left(\frac{\operatorname{dist}(\varphi(z), \partial \varphi(\mathbf{D}))}{\operatorname{dist}(\varphi(z), \partial \mathbf{D})}\right)^{q} .
$$


So, let us define

$$
A_{\delta}:=\left\{z \in \mathbb{D}: \frac{\operatorname{dist}(\varphi(z), \partial G)}{\operatorname{dist}(\varphi(z), \partial \mathbb{D})} \geqslant \delta\right\} .
$$

Then on account of what was just said, we get that

$$
A_{\delta} \subseteq{ }^{(\delta / 2)^{q}} \Omega_{\varphi_{,\left(\varphi^{\prime}\right) q}^{v_{q}}} .
$$

This means that we can work with the sets $A_{\delta}$ instead of ${ }^{\varepsilon} \Omega_{\varphi,\left(\varphi^{\prime}\right)^{q}}^{v_{q}}$ which gives us the great advantage of working with the image of $\varphi$ directly (see Figure 2 (a) for a sketch of $\left.\varphi\left(A_{1 / 7}\right)\right)$.
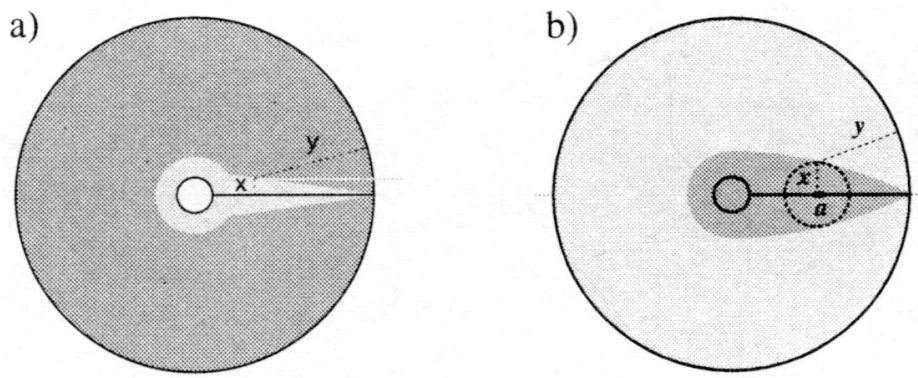

Figure 2: In this figure, referring to Example 5.2, we have chosen $q=1 / 10$ and $r_{0}=1 / 10$ for visibility (see Remark 3.3). In (a) the set $\varphi\left(A_{1 / 7}\right)$ is shown. That is, the ratio between $x$ and $y$ is 1 to 7 . In (b) a pseudo-hyperbolic neighbourhood with radius $R_{q}^{-1}$ of the interval $[0,1)$ is shown $\left(R_{q}^{-1}=0.2405\right)$. As one can clearly see, $\varphi\left(A_{1 / 7}\right) \cap \overline{D(a, 0.24)} \neq \emptyset$ for all $a \in[0,1)$.

For any $r \in(0,1)$ and any $b \in \mathbb{D}$, with $\operatorname{Re}(b) \geqslant 0$ and $\operatorname{Im}(b)>0$, we can find $a \in[0,1)$ such that $\operatorname{Re}\left(c_{E}(b, r)\right)=\operatorname{Re}\left(c_{E}(a, r)\right)$. A straightforward calculation shows that

$$
\operatorname{Im}\left(c_{E}(b, r)\right)+r_{E}(b, r) \geqslant r_{E}(a, r) .
$$

Hence, due to the symmetry of $\varphi\left(A_{\delta}\right)$ (see Figure $2(\mathrm{a})$ ) and the preceding discussion, it suffices to show that there exists $\delta>0$ and $r \in\left(0, R_{q}^{-1}\right)$ such that $\varphi\left(A_{\delta}\right) \cap \overline{D(a, r)} \neq \emptyset$ for all $a \in[0,1)$.

Consider now the function

$$
f(a, r):=\frac{r_{E}(a, r)}{1-\left(r_{E}(a, r)^{2}+c_{E}(a, r)^{2}\right)^{1 / 2}}, \quad \text { where } a \in[0,1) .
$$

A straightforward calculation shows that for any fixed $r$, we have that

$$
\inf _{a \in[0,1)} f(a, r)=\min \left\{f(0, r), \lim _{a \rightarrow 1} f(a, r)\right\}=\min \left\{\frac{r}{1-r}, \frac{2 r}{1+r^{2}}\right\}=: \gamma_{r} .
$$


In figure 2 (b) one can see the pseudo-hyperbolic neighbourhood with radius $R_{q}^{-1}$ of the interval $[0,1)$, or more precisely, the set

$$
B_{r}:=\bigcup_{a \in(0,1)} \overline{D(a, r)}, \quad \text { with } r=R_{q}^{-1} .
$$

The ratio between the numerator and the denominator of $f\left((1 / 2), R_{q}^{-1}\right)$ (denoted by $x$ and $y$, respectively) can also be seen.

Thus, by fixing $r \in\left(r_{0}, R_{q}^{-1}\right)$ and choosing $\delta<\gamma_{r}$ we get that $\varphi\left(A_{\delta}\right) \cap \overline{D(a, r)} \neq \emptyset$ for all $a \in[0,1)$.

EXAMPLE 5.3. Let $G_{0}=\mathbb{D}$. For $n \in \mathbb{N}$, we consider the following recursive construction method. Construct $G_{n}$ by deleting finitely many radial slits of length $2^{-n}$ (with one endpoint on $\partial \mathbb{D})$ from $G_{n-1}$ so that $\operatorname{dist}\left(a, \partial G_{n}\right) \leqslant 3^{-n}$ for every $a \in G_{n}$ with $|a| \geqslant 1-2^{-n}$. Let $G=\bigcap_{n=0}^{\infty} G_{n}$ and let $\varphi$ be the univalent mapping that maps $\mathbb{D}$ onto $G$. Then $W_{\varphi,\left(\varphi^{\prime}\right) \varphi}$ does not have closed range on $H_{v_{q}}^{\infty}$ even though $\overline{\varphi(\mathbb{D})}=\overline{\mathbb{D}}$ (see Figure 3).

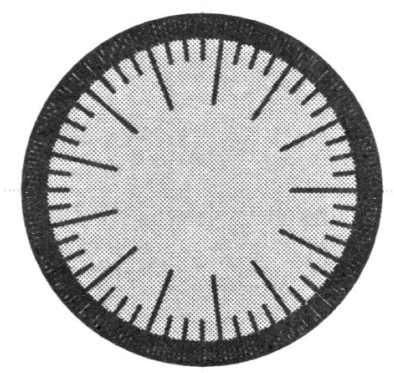

Figure 3: $G_{3}$ in Example 5.3. When the slits are evenly spaced as above it suffices to delete 13 slits from $G_{0}, 13 \cdot 3$ slits from $G_{1}, 13 \cdot 3 \cdot 3$ slits from $G_{2}$ and finally $13 \cdot 3 \cdot 3 \cdot 2^{n-2}$ slits from every $G_{n}$ with $n \geqslant 3$.

Proof: From the construction of $G$ it is easy to see that for any $n \in \mathbb{N}$,

$$
\frac{\operatorname{dist}(\varphi(z), \partial G)}{\operatorname{dist}(\varphi(z), \partial \mathbb{D})} \leqslant \frac{3^{-n}}{2^{-n-1}} \quad \text { whenever } \quad 1-2^{-n} \leqslant|\varphi(z)| \leqslant 1-2^{-n-1} .
$$

Again, as in Example 5.2, we shall use Koebe's one-quarter theorem, to get that

$$
\lim _{|\varphi(z)| \rightarrow 1}\left|\tau_{\varphi,\left(\varphi^{\prime}\right) q}^{v_{q}}(z)\right| \approx \lim _{|\varphi(z)| \rightarrow 1}\left(\frac{\operatorname{dist}(\varphi(z), \partial G)}{\operatorname{dist}(\varphi(z), \partial \mathbb{D})}\right)^{q} \leqslant \lim _{n \rightarrow \infty} 2^{q}\left(\frac{2}{3}\right)^{n q}=0 .
$$

Corollary 3.12 finishes the proof (see also Remark 3.13 and Remark 3.14). 


\section{The Bloch-TYPE SPACES}

In this section we shall show that Theorem 3.7, Corollary 3.12, Theorem 4.1 and Corollary 3.8 are also true for the composition operator $C_{\varphi}$ on the Bloch-type space $\mathcal{B}^{q}$ with some minor modifications (see Corollary 6.2).

We begin by noticing that for any $a \in \mathbb{D}$ we have that $C_{\sigma_{a}}$ is bounded on $\mathcal{B}^{q}$. Hence, we may always assume that $\varphi(0)=0$. Next we shall prove an intuitively evident, but important lemma, which we state formally for completeness.

LEMMA 6.1. $C_{\varphi}$ is bounded on $\mathcal{B}^{q}$ if and only if $W_{\varphi, \varphi^{\prime}}$ is bounded on $H_{v_{q}}^{\infty}$. Similarly, $C_{\varphi}$ has closed range on $\mathcal{B}^{q}$ if and only if $W_{\varphi, \varphi^{\prime}}$ has closed range on $H_{v_{q}}^{\infty}$.

Proof: Consider the mapping $S_{q}: \mathcal{B}^{q} \rightarrow H_{v_{q}}^{\infty}$, given by $S_{q} f=f^{\prime}$. One can easily verify that $S_{q}$ is an onto isometry and since $C_{\varphi}=S_{q}^{-1} \circ W_{\varphi, \varphi^{\prime}} \circ S_{q}$, we are finished.

Corollary 6.2. (Summary for the Bloch-type spaces)

(a) If $C_{\varphi}: \mathcal{B}^{q} \rightarrow \mathcal{B}^{q}$ has closed range, then there exists $\varepsilon>0$ such that

$$
\varphi\left({ }^{\varepsilon} \Omega_{\varphi, \varphi^{\prime}}^{v_{q}}\right) \cap \overline{D(a, 1-\varepsilon)} \text { for all } a \in \mathbb{D} \text {. }
$$

(b) Let $R_{q}$ be the constant in Lemma 3.6. If there are $r \in\left(0, R_{q}^{-1}\right)$ and $\varepsilon>0$ such that

$$
\varphi\left({ }^{\varepsilon} \Omega_{\varphi, \varphi^{\prime}}^{v_{q}}\right) \cap \overline{D(a, r)} \text { for all } a \in \mathbb{D},
$$

then $C_{\varphi}: \mathcal{B}^{q} \rightarrow \mathcal{B}^{q}$ has closed range.

(c) If $C_{\varphi}: \mathcal{B}^{q} \rightarrow \mathcal{B}^{q}$ has closed range, then there exists $\varepsilon>0$ such that

$$
\overline{\varphi\left(\varepsilon \Omega_{\varphi, \varphi^{\prime}}^{v_{q}}\right)} \supseteq \partial \mathbb{D}
$$

(d) Let $\varphi$ be univalent and assume that $q \geqslant 1$. Then $C_{\varphi}$ has closed range on $\mathcal{B}^{q}$ if and only if there exists $\varepsilon>0$ such that

$$
\varphi\left(\Omega_{\varphi, \varphi^{\prime}}^{v_{\varphi}}\right) \cap \overline{D(a, 1-\varepsilon)} \text { for all } a \in \mathbb{D}
$$

(e) Let $\varphi$ be univalent and assume that $\varphi^{\prime} \in H^{\infty}$. Then $C_{\varphi}$ has closed range on $\mathcal{B}^{q}$ (for any $q$ ) if and only if there exists $\varepsilon>0$ such that

$$
\varphi\left({ }^{\varepsilon} \Omega_{\varphi, \varphi^{\prime}}^{v_{1}}\right) \cap \overline{D(a, 1-\varepsilon)} \neq \emptyset \text { for all } a \in \mathbb{D}
$$

Proof: A direct consequence of Lemma 6.1 as well as Theorem 3.7 (a), Theorem 3.7 (b), Corollary 3.12 (see also Remark 3.13), Theorem 4.1 and Corollary 4.6, respectively.

REMARK 6.3. By choosing $q=1$ in Corollary 6.2 we get the corresponding results for the Bloch space obtained in $[13,14]$. Note that the constant $R_{q}$ in Lemma 3.6 becomes 
18.22 which is expectedly somewhat greater than 3.31 calculated specifically for the Bloch space (see [13, Theorem 1 and Theorem 2]). Actually, by a slight modification of their proof, the constant 3.31 can be reduced to 1.93 (by applying the same ending as in the proof of Lemma 3.6).

EXAMPLE 6.4. $\overline{\varphi(\mathbb{D})}=\overline{\mathbb{D}}$ is neither necessary nor sufficient for $C_{\varphi}$ to have closed range on $\mathcal{B}$.

Proof: Follows directly from Lemma 6.1 and by choosing $q=1$ in Example 5.2 and Example 5.3.

\section{REFERENCES}

[1] K.D. Bierstedt, J. Bonet and J. Taskinen, 'Associated weights and spaces of holomorphic functions', Studia Math. 127 (1998), 137-168.

[2] J. Bonet, 'Weighted spaces of holomorphic functions and operators between them', in Seminar of Mathematical Analysis (Malaga/Seville, 2002/2003), Colecc. Abierta 64 (Univ. Sevilla Secr. Publ., Seville, 2003), pp. 117-138.

[3] J. Bonet, P. Domański and M. Lindström, 'Essential norm and weak compactness of composition operators on weighted Banach spaces of analytic functions', Canad. Math. Bull. 42 (1999), 139-148.

[4] J. Bonet, P. Domański and M. Lindström, 'Pointwise multiplication operators on weighted Banach spaces of analytic functions', Studia Math. 137 (1999), 177-194.

[5] J. Bonet, P. Domański, M. Lindström and J. Taskinen, 'Composition operators between weighted Banach spaces of analytic functions', J. Austral. Math. Soc. Ser. A 64 (1998), 101-118.

[6] P. Bjørstad and E. Grosse, 'Conformal mapping of circular arc polygons', SIAM J. Sci. Statist. Comput. 8 (1987), 19-32.

[7] K. Cichoń and K. Seip, 'Weighted holomorphic spaces with trivial closed range multiplication operators', Proc. Amer. Math. Soc. 131 (2002), 201-207.

[8] M.D. Contreras and A.G. Hernandez-Díaz, 'Weighted composition operators in weighted Banach spaces of analytic functions', J. Austral. Math. Soc. Ser. A 69 (2000), 41-60.

[9] C.C. Cowen and B.D. MacCluer, Composition operators on spaces of analytic functions (CRC Press, Boca Raton, 1995).

[10] P. Domański and M. Lindström, 'Sets of interpolation and sampling for weighted Banach spaces of holomorphic functions', Ann. Polon. Math. 79 (2002), 233-264.

[11] T.A. Driscoll and L.N. Trefethen, Schwarz-Christoffel mapping (Cambridge University Press, Cambridge, 2002).

[12] J.B. Garnett, Bounded analytic functions (Academic Press, New York, 1981).

[13] P. Ghatage, J. Yan and D. Zheng, 'Composition operators with closed range on the Bloch space', Proc. Amer. Math. Soc. 129 (2001), 2039-2044.

[14] P. Ghatage, D. Zheng and N. Zorboska, 'Sampling sets and closed range composition operators on the Bloch space', Proc. Amer. Math. Soc. 133 (2005), 1371-1377.

[15] L.H. Howell, 'Numerical conformal mapping of circular arc polygons', J. Comput. Appl. Math. 46 (1993), 7-28. 
[16] H. Hedenmalm, B. Korenblum and K. Zhu, Theory of Bergman spaces (Springer-Verlag, New York, 2000).

[17] M. Lindström and N. Palmberg, 'Spectra of composition operators on BMOA', Integral Equations Operator Theory 53 (2005), 75-86.

[18] D. Luecking, 'Inequalities on Bergman spaces', Mlinois J. Math. 25 (1981), 1-11.

[19] W. Lusky, 'On weighted spaces of harmonic and holomorphic functions', J. London Math. Soc. 51 (1995), 309-320.

[20] S. Ohno, K. Stroethoff and R. Zhao, 'Weighted composition operators between Bloch-type spaces', Rocky Mountain J. Math. 33 (2003), 191-215.

[21] S. Ohno and H. Takagi, 'Some properties of weighted composition operators on algebras of analytic functions', J. Nonlinear Convex Anal. 2 (2001), 369-380.

[22] C. Pommerenke, Boundary behaviour of conformal maps (Springer-Verlag, Berlin, 1992).

[23] R.C. Roan, 'Composition operators on $H^{p}$ with dense range', Indiana Univ. Math. J. 27 (1978), 159-162.

[24] S. Shimorin, 'Weighted composition operators associated with conformal mappings', in Quadrature Domains and Their Applications (The Harold S. Shapiro Anniversary Volume), Oper. Theory Adv. Appl. 156 (Birkhäuser, Basel, 2005), pp. 217-237.

[25] W. Smith, 'Composition operators between Bergman spaces and Hardy spaces', Trans. Amer. Math. Soc. 348 (1996), 2331-2348.

[26] A.L. Shields and D.L. Williams, 'Bounded projections, duality and multipliers in spaces of analytic functions', Trans. Amer. Math. Soc. 162 (1971), 287-302.

[27] A.L. Shields and D.L. Williams, 'Bounded projections and the growth of harmonic conjugates in the disc', Michigan Math. J. 29 (1982), 3-25.

[28] K. Zhu, Operator theory in function spaces (Marcel Dekker Inc., New York, 1990).

Department of Mathematics

Åbo Akademi University

Fänriksgatan 3 B

FIN-20500 Åbo

Finland

e-mail: niklas.palmberg@abo.fi 